\title{
Diogene Laerzio e Clemente Alessandrino nel contesto di un dibattito culturale comune
}

\author{
ILARIA RAMELLI \\ [Università Cattolica del Sacro Cuore, Milano]
}

\begin{abstract}
RESUMEN.
II Presente articolo analizza i parallelismi tra le Vitae

Philosophorum di Diogene Laerzio e

gli Stromata di Clemente

Alessandrino e pone entrambi nel contesto di un dibattito culturale comune relativo alle origini della filosofia.
\end{abstract}

PAROLE CHIAVE:

Clemente di Alessandria, Cultura greca e cristianesimo, Diogene Laerzio, Filosofia greca, Origini della filosofia

\begin{abstract}
.
The present article analyses the parallels between Diogenes Laertius' Vitae Philosophorum and Clement of Alexandria's Stromata, and sets them both in the context of a common cultural debate concerting the origins of philosophy.
\end{abstract}

KEY WORDS:

Clement of Alexandria, Diogenes

Laertius, Greek culture and

Christianity, Greek philosophy,

Origins of philosophy.

In un articolo precedente, partendo da alcuni spunti di L. Canfora ${ }^{1}$, ho esaminato la presenza di riscontri tra le Vitae philosophorum di Diogene Laerzio e l'Oratio ad Graecos di Taziano, nella quale non solo è possibile individuare numerosi parallelismi con il testo laerziano ${ }^{2}$, ma si può forse anche supporre una citazione dell'opera di Diogene da parte dell'apologista cristiano, in Orat. 37, laddove è citato un Naîtos che avrebbe tradotto in greco le opere -comprensive anche della storia del ratto di Europa e della relativa cronologia, posta a confronto con quella ebraica ${ }^{3}$ - di tre scrittori fenici, Teodoto, Issicrate e Moco, e che è designato come colui che "ha descritto minutamente le vite dei filosofi»: secondo Canfora, questo nome saitos, lezione filologicamente tormentata, andrebbe emendato proprio in

- Clemente di Alessandria e Diogene Laeizio, in Storia poesia e pensiero nel mondo antico. Studi in onore di Marcello Gigante, Napoli 1992, pp. 79-81.

2 I. Ramelli, "Diogene Laerzio e i Cristiani: conoscenza e polemica con Taziano e con Clemente Alessandrino?», Espacio, Tiempo y Forma, Serie II, 15 (2004), pp. 27-42; EAD. Diogene Laerzio: Vite dei filosofi, introduzione, traduzione, commento, con apendice degli Estratti Bizantini, in coll. con G. GIRGENTI, Milano 2004. Cfr. anche G.F. Hawthorn, Tatian and His Discourse to the Greeks, "Harvard Theological Review” 57 (1964), pp. 161-188.

3 L'attenzione per queste tematiche si situa nel contesto della diatriba sulla maggiore antichità della sapienza greca o di quella 'barbara', e segnatamente di quella ebraico-cristiana di cui riparleremo e che coinvolgeva sia Diogene sia Taziano sia Clemente. 
$\Lambda a \dot{\varepsilon} \rho T\left(\alpha \varsigma_{6}^{4}\right.$. Tanto più interessante si rivela l'ipotesi di un milieu culturale comune a questi autori e di una polemica in atto tra di loro, alla luce dell'impiego in Diogene di un termine tipicamente cristiano come $\dot{\varepsilon} \lambda \varepsilon \eta \mu$ oviv' in Vit. phil. $V 17$ e dell'ipotesi di una localizzazione tanto di Diogene quanto di Clemente nell'Alessandria tra II e III secolo ${ }^{5}$, dove Clemente dirigeva il $\Delta і \delta a \sigma \kappa a \lambda \varepsilon i(1), 6$.

Una volta compiuta, nel mio lavoro precedente, la disamina sistematica delle convergenze con Taziano, la cui Oratio ad Graecos presenta infatti la maggiore frequenza di passi paralleli con Clemente Alessandrino e con Diogene Laerzio ${ }^{7}$, è bene, dunque, analizzare sistematicamente tutti i passi paralleli tra Diogene e Clemente Alessandrino ${ }^{8}$.

L'esordio stesso dell'opera di Diogene Laerzio è dedicato alla discussione del problema delle origini della filosofia: era dibattuta all'epoca la questione se il pensiero filosofico fosse nato presso i $\mathrm{Greci}$, come sostiene lo stesso Diogene, oppure presso i barbari, come afferma Taziano. Diogene apre la sua opera (Vitae, I 1) così: "L'attività della filosofia, alcuni dicono che abbia avuto origine dai barbari. Ci sono stati, infatti, presso i Persiani i Magi, presso i Babilonesi o gli Assiri i Caldei, e i Gimnosofisti presso gli Indiani, e presso i Celti e i Galli i cosiddetti Druidi e Semnote ${ }^{9}$, come afferma Aristotele nel suo trattato Sui Magi ${ }^{10}$ e Sozione

${ }^{4}$ L. CANFORA, Da Socrate a Epicuro, l'Occidente abita qui, recensione all'edizione Marcovich, "Corriere della Sera", 22. IV. 2001, p. 29; la proposta è analizzata in Ramelli, Diogene e i Cristiani, cit.

Ipotesi vagliata nel mio articolo citato Diogene e i Cristiani. Su Clemente segnalo solo alcuni studi fondamentali: C. Mondésert, Clément d'Aléxandrie, Paris 1944; P. NAUTIN, Lettres et écrivains chrétiens des Ilème et Illème siècles, Paris 1961, pp. 138-141; A. MEHAT, Études sur les "Stromates" de Clément, Paris 1966; S. LILLA, Clement of Alexandria, A Study in Christian Platonism and Gnosticism, Oxford UP - London 1971; R. MORTLEY, Connaissance religieuse et herméneutique chez Clément, Leiden 1973; C. MORESCHINI E. NoRELLI, Storia della letteratura cristiana antica greca e latina, I, Brescia 1995, pp. 360-377; S. SAID - M. TREdE - A. LE BOULLUEC, Histoire de la littérature grecque. Paris 1997, pp. 585-588; Clément d'Aléxandrie, Les Stromates, Stromate VI, intr. par P. Descourtieux, Paris 1999, Sources Chrétiennes 446, pp. 7-11. Ulteriore, ampia documentazione nel mio Mysterion negli Stromatêis di Clemente Alessandrino, in VV. AA., II mistero nella carne, II, ac. di A.M. Mazzantı, Bologna 2005, in pubblicazione.

6 Cfr. C. Bardy, Aux origines de l'école d'Aléxandrie, "Recherches de Sciences Religieuses" 27 (1937), pp. 65-90; Eiusd. Pour l'histoire de l'école d'Aléxandrie, "Revue Biblique» 1942 [= Vivre et Penser, II ser.], pp. 80-109; A. VACCARI, Primo abbozzo di università cristiana, in Scritti di Erudizione e Filologia, Roma 1952, pp. 73-96; F. PERICOLI RIDOLFINI, Le origini della scuola di Alessandria, "Rivista degli Studi Orientali" 37 (1962), pp. 211-230.

7 Cfr. I'apparatus fontium di Tatiani Oratio ad Graecos, ed. M. Marcovich, Berlin-New York 1995, Patristische Texte und Studien 43-44, pp. 7-75.

8 Adotto per gli Stromata, con alcuni interventi, la traduzione Clemente Alessandrino. Stromati: note di vera filosofia, tr. G. Pini, Milano 1985, Letture cristiane delle origini 20; cfr anche, con introduzione e bibl. cui rinvio, Clemente Alexandrino. Stromatéis: memorias gnósticas de verdadera filosofia, tr. D. Mayor, Abadia de Silos 1993, Studia Silensia 16. Edizione critica degli Stromata: Clemens Alexandrinus, II, Stromata, Buch 1VI, ed. O. Stählin, nuova ed. di L. Fruchtel, $4^{\wedge}$ ed. a cura di U. Treu, Berlin 1985, GCS. Per il titolo si veda L. Alfonsi, Sul titolo 'Stromati', “Sileno" 1 (1975), pp. 175-176. Le versioni di Diogene invece sono mie, condotte su Diogenis Laertii Vitae Philosophorum, ed. M. Marcovich, I; II (Excerpta Byzantina), Leipzig 1999.

${ }_{9}$ Cfr. Steph. Byz. s.v. $\Delta$ puínat. Per i Semnotei, cfr. Alex. Polyhist. FGH 273 F 18.

$10 \mathrm{Fr} .661$ Gigon; è un trattato pseudo-aristotelico che presentava il persiano Zoroastro come inventore della sapienza, e i cui frammenti sono raccolti da O. GIGON, Aristotelis Opera, III, Librorum deperditorum fragmenta, Berlin 1987, frr. 660-665. Cfr. anche fr. 35 Rose $^{3}$. Sui Magi si veda ad es. 
nel ventitreesimo libro della Successione (dei filosofi) ${ }^{11}$. E che Oco era fenicio, Zalmoxi tracio e Atlante libico". Ora, Clemente Alessandrino, Strom. I 15, 71, 3-4, mostrando come la filosofia sia pervenuta ai barbari prima che ai Greci, enumera i filosofi barbari in una lista molto simile a quella laerziana: «La filosofia... fiori presso i barbari... solo più tardi giunse anche in Grecia. Iniziatori ne furono i profeti egizi, i Caldei tra gli assiri, i Druidi tra i Galli, i Samanei nella Battriana, i filosofi tra i Celti, i Magi persiani... i Gimnosofisti indiani... distinti in Sarmani e Brahmani „2. Poco dopo, in Strom. I 16, 74, 1, 1 Clemente presenta Atlante, citato anche da Diogene nel passo appena visto, come l'inventore della navigazione, nel contesto della scoperta di "quasi ogni arte", attribuita ai barbari ${ }^{13}$. Anche il mitico Zalmoxi, pure citato nel passo laerziano, è noto a Clemente, che in Strom. IV 8, 57, 2 lo presenta come un barbaro pitagorico: «I Geti, popolo barbaro non digiuno da filosofia, scelgono un ambasciatore da mandare come eroe a Zalmoxis ogni anno: questo Zalmoxis era in realtà uno degli allievi di Pitagora». Clemente riflette la notizia di Erodoto, il quale in Hist. IV 94-96 narra che Zalmoxi era una divinità venerata dai Geti, suggerendo in senso evemeristico che fosse un servo tracio di Pitagora che si presentò come divinità ai suoi connazionali ${ }^{14}$. Lo stesso Diogene, citando espressamente quale fonte Erodoto, riparla di Zalmoxi in VIII 2, con una metatesi nella grafia del nome, dichiarandolo di stirpe getica e servo di Pitagora: "(Pitagora) aveva anche alcuni fratelli... e uno schiavo, Zamolxi, al quale i Geti offrono sacrifici rituali, ritenendolo Crono, come afferma Erodoto".

Subito dopo la disamina degli argomenti degli avversarî, secondo cui la filosofia sarebbe nata tra i barbari, il Laerzio oppone a questi la propria tesi, secondo cui la filosofia e la civiltà stessa avrebbero avuto origine dai Greci: "Ma essi mancano di riconoscere le felici realizzazioni dei Greci, dalle quali non solo ebbe origine la filosofia, ma anche il genere umano, attribuendole ai barbari. Ecco, per esempio, presso gli Ateniesi ci fu Museo; presso i Tebani, poi, Lino...” (Vitae, I 3). Invece Clemente Alessandrino, Strom. I 15,67, 1, elogia Platone poiché «ritiene che vi siano anche fra i barbari dei filosofi, contrariamente ad Epicuro, per il quale solo i Greci sono capaci di filosofare».

1 Fr. 35 Wehrli.

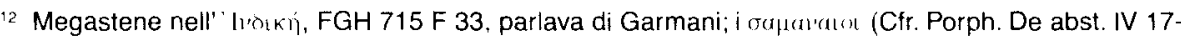
18) sarebbero monaci buddhisti secondo A. Diehle, Neues zu Thomas-Tradition, «Jahrbuch für Antike und Christentum" 6 (1973), pp. 54-70; Eiusd. Indische Philosophie bei Clemens, ibid. 1974, pp. 60-70; cfr. I. RAMELLI, La missione di Panteno in India: alcune osservazioni, in La diffusione dell'eredità classica nell'età tardoantica e medievale. Filologia, Storia, Dottrina, Atti del Seminario Nazionale di Studio, Napoli-Sorrento 29-31 ottobre 1998, a c. di C. Baffioni, Alessandria 2000, pp. 95-106; C. DOGNINI - I. RAMELLI, Gli Apostoli in India nella Patristica e nella letteratura sanscrita, Milano 2001; I. RAMELLI, Note sulle origini del Cristianesimo in India, "Studi Classici e Orientali».

13 Secondo Cicerone, Atlante era esperto dei fenomeni celesti (Tusc. V 3, 8). Cfr. 68 A 55 D. $-\mathrm{K}$

${ }^{14}$ L'attenzione di Clemente verso questo personaggio si riflette anche in Origene, C. Cels. III 54 
In effetti la posizione di Clemente rispetto alle origini della sapienza ${ }^{15}$, in parte convergente con quella di Giustino ${ }^{16}$, è quella di fare risalire la sapienza non ai Greci, ma nemmeno ai barbari in sé, bensi a Dio-logos ${ }^{17}$ : "Anche se risalgo a Pitagora, Ferecide, Talete, mi soffermo a cercarne i maestri; se tu me li trovi negli Egiziani, Indiani, Babilonesi, nei Magi stessi, non esiterò ad andare in cerca del maestro di questi; ti ricondurrò fino alla prima generazione umana, e ancora di lì comincerò a cercare: chi è maestro? Nessuno degli uomini potrebbe avere originato la filosofia, poiché

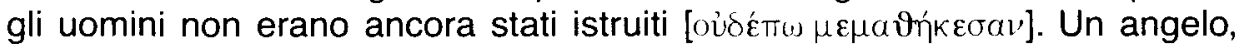
allora? Nemmeno... Uno, l'ingenerato, per mezzo del quale tutto fu fatto e senza di lui niente fu fatto... poiché è chiamato 'sapienza di tutti i profeti', è egli il maestro di tutti gli esseri generati» (Strom. VI 7, 57, 3-4) ${ }^{18}$. Clemente riconosce tranquillamente la scoperta di scienze particolari ai pagani e ai barbari, come la geometria agli Egizi, I'astronomia ai Babilonesi, la medicina ai Traci (Protrept. VI 70, 1; Strom. 1 14-16). Ma d'altra parte non esita ad accusare di plagio i filosofi greci in campo etico e teologico (Protr. VI 70, 1-5; Strom. I 22, 150; 25, 105); è noto che presenta Platone come emulo di Mosè (Paed. III 11, 54, 2) e che lo loda più volte per avere ammesso di avere importato dai barbari alcune concezioni filosofiche (Strom. I 15, 66-69).

II contesto è quello della diatriba tra le posizioni greche pagane e gli Apologisti cristiani, che rivendicano alla tradizione ebraico-cristiana la priorità e la conformità all'antica sapienza originaria e incorrotta, vera, da cui il sapere ellenico, pagano, si sarebbe discostato. Questa concezione affonda le radici nell'idea, sostenuta dagli autori giudaici, che la cultura greca sia più recente $e$ derivi da quella ebraica, corrompendola ${ }^{19}$. Di speciale interesse sembra Aristobulo, il quale nel II sec. a.C. riteneva che la Scrittura ebraica avesse ispirato Pitagora, Orfeo, Arato, Omero, Esiodo, Lino, Platone e Aristotele (ap. Eus. Praep. Ev. XIII 12, 663 D - 668 B), e che propugnava un'interpretazione non letterale della Scrittura. Secondo Giuseppe Flavio, Platone, Pitagora e i più antichi dei Greci avrebbero ripreso le Scritture ebraiche (part. Adv. Apion. I

15 Cfr. E. Molland, Clement of Alexandria on the Origin of Greek Philosophy, "Symbolae Osloenses" 15-16 (1936), pp. 57-85; J. WASZINK, Observations on the Appreciation of the "Philosophy of the Barbarians", in Mélanges Chr. Mohrmann, Utrecht 1963, pp. 41-56; G.R. Boys-Stones, Post-Hellenistic Philosophy. A Study of its Development from the Stoics to Origen, Oxford, University Press, 2001, pp. VII; 188-194.

16 Su cui cfr. lo stesso Boys-Stones, Post-Hellenistic Philosophy, pp. 184-188 con bibliogr. cui va aggiunto G. GIRGENTI, Giustino martire. II primo cristiano platonico, Milano 1995. Sulla posizione di Clemente verso la filosofia greca sono ancora importanti Th. CAMELOr, Clément et l'utilisation de la philosophie grecque, “Recherches de Sciences Religieuses» 21 (1931), pp. 541-569; Eiusd. Les idées de Clément sur l'utilisation des sciences et de la littérature profane, ibid. pp. 38-66; J. MUCKLE, Clemens on Philosophy as a Divine Testament for the Greeks, "Phoenix" 5 (1951), pp. 79-86

17 Cfr. per la dottrina del logos che da Giustino passa a Clemente e poi a Origene, per le sue ascendenze giovannee e classiche:W. KELBER, Die Logoslehre von Heraklit bis Origen, Stuttgart 1958; ulteriore documentazione nel mio cit. Mysterion negli Stromateis e anche nel mio II multiforme uso di Mysterion in Giustino, ibidem.

$18 \mathrm{Cfr}$. ibid. 18, 166, 4: "Solo da Dio è insegnata la nostra sapienza, dalla quale dipendono tutte le sorgenti di sapienza, quelle almeno che mirino a raggiungere la verità". Si vedano $E$. FASCHER, Der göttlicher Lehrer bei Clemens, in Studien zum Neuen Testament und Patristik E. Klostermann dargebracht, Berlin 1962, pp. 193-207; L. FARINELLI, Filosofia e rivelazione in Clemente, "Filosofia e Vita" 6 (1965), pp. 227-243

19 Si veda la disamina di questa tradizione apologetica ebraica in Boys-Stones, Post-Hellenistic Philosophy, pp. 76-98. 
162; II 168), mentre i veri sapienti sarebbero gli antichi Patriarchi (Ant. lud. I 105-106). Anche per Filone, filosofi come Eraclito, Pitagora, Aristotele e Zenone ${ }^{20}$ dovrebbero la loro sapienza a quella giudaica.

Tra i Greci che, come afferma Diogene, furono dunque i fondatori della filosofia, un ruolo particolare spetta a Pitagora, il quale avrebbe addirittura inventato, applicandolo per primo a se stesso, il nome di pidófopos, in base al pensiero che solo Dio è, propriamente, sapiente (oopós) mentre un essere umano si limita ad aspirare alla sapienza, ad essere "amante della sapienza», ossia, appunto, filo-sofo. Diogene (Vitae, I 12) riferisce come segue:

A dare per primo il nome alla filosofia e a chiamare se stesso filosofo fu Pitagora, discutendo a Sicione con Leone, il tiranno di Sicione o di Fliunte, secondo quanto afferma Eraclide Pontico nell'opera Sull'inanimato: nessuno, infatti, a suo avviso è sapiente se non $\mathrm{Dio}^{21}$. Troppo precipitosamente, infatti, si dava il nome di sapienza (sophía) e si chiamava sapiente (sophós) chi la professa, il quale sarebbe giunto alla conoscenza esatta nell'alto dell'anima, mentre filosofo è colui che aspira alla sapienza. Ora, i sapienti erano chiamati anche sofisti; e non solo <costoro>, ma anche i poeti erano detti tali, come anche Cratino, nei suoi Archilochi ${ }^{22}$, lodando i seguaci di Omero e di Esiodo, li chiama così.

Anche in questo caso troviamo un duplice riscontro con Clemente: in Strom. I 14, 61, 4: «Pitagora... per primo chiamò se stesso 'filosofo'»; IV 3, 9, 1: "Pitagora... dice che soltanto Dio è sapiente... e che egli stesso è amante della sapienza». Clemente si occupa di Pitagora ... Quanto alla menzione di Cratino da parte di Diogene, anche Clemente in Strom. I 3, 24, 1-2 cita lo stesso passo: "Cratino negli Archilochi, dopo aver fatto un elenco di poeti, dice: 'Che sciame di sofisti avete trovato!'».

La ripartizione generale della filosofia nelle grandi scuole italica, iniziata da Pitagora, e ionica, iniziata da Talete, è identica in Diogene (Vit. I 13-14) e in Clemente, che aggiunge anche quella eleatica, da Senofane (Strom. I 14, $62,1)$. Simili sono anche gli schemi di successioni di filosofi proposti da entrambi gli autori.

Nella trattazione sistematica dei filosofi entro le Vitae philosophorum, la prima scheda bio-dossografica è dedicata da Diogene a Talete, che inaugura non solo la scuola filosofica ionica, ma al contempo anche la serie dei sette Sapienti. Nelle Vitae, I 22 Talete è ricondotto non solo a origini milesie, ma anche, in alternativa, a stirpe fenicia: "Ebbene, Talete, come dicono Erodoto, Duride e Democrito, aveva come padre Esamia e come madre Cleobuline, della stirpe dei Telidi, che sono fenici, nobilissimi tra i discendenti di Cadmo

20 I passi sono raccolti da Boys-Stones, Post-Hellenistic Philosophy, p. 92.

21 Fr. 87 Wehrli. Cfr. lamblich. Vita Pythag. 58-59; Diog. Laërt. VIII 8; Cic. Tusc. V 8; Diod. Sic. Bibl. Hist. $X 10,1$.

22 Fr. 2 Kassel-Austin (PCG IV). 
e di Agenore»23. Come, invece, sostengono i più, era un nativo di Mileto, e di stirpe illustre". Analoga alternativa è proposta da Clemente in Strom. I 14, 62, 3: «Pitagora era un barbaro. Ma anche Talete, come raccontano Leandro ed Erodoto, era fenicio; secondo altri, invece, milesio". Sempre a proposito di Talete, una sua celebre definizione del divino si trova identica in Diogene (I 36: “'Che cosa è il divino?' 'Quello che non ha né inizio né fine'») e in Clemente Alessandrino, Strom. V 14, 96, 4: “Quando gli fu chiesto che cosa sia la divinità, Talete rispose: 'Ciò che non ha né principio né fine'»; Clemente riconduce questa sentenza alla verità cristiana (ibid.) ${ }^{24}$. L'attribuzione della previsione di un'eclissi a Talete è comune a Diogene e a Clemente, che citano le medesime fonti, Eudemo ed Erodoto: il primo in I 23 ricorda: "Sembra, poi, secondo alcuni, che sia stato il primo a studiare astronomia e a predire le eclissi di sole e i solstizî, come dice Eudemo nella sua Storia degli studi di astronomia: per questo lo ammirano sia Senofane sia Erodoto. Gli rendono testimonianza, poi, sia Eraclito sia Democrito»25. Clemente in Strom. I 14, 65, 1 riferisce parimenti: «Quanto a Talete, Eudemo nella sua Storia degli studi di astronomia afferma che predisse l'eclissi di sole avvenuta il giorno in cui combatterono i Medi e i Lidi, allorché regnava sui Medi Ciassarre padre di Astiage e sui Lidi Aliatte, padre di Creso. Concorda con Eudemo anche Erodoto nel I libro. Si tratta dunque degli anni intorno alla $50^{\text {a }}$ Olimpiade».

Nel contesto delle varie liste dei Sette Sapienti, Diogene in I 41 termina con: "Alcuni aggiungono, poi, Acusilao, figlio di Caba o di Scabra, di Argo" ${ }^{26}$; Clemente in Strom. | 14, 59, 5, dopo avere fornito anch'egli liste discordanti dei Sette, conclude con: "Altri poi inclusero Acusilao di Argo fra i Sette Sapienti». Un'altra piccola convergenza riguarda un altro dei suddetti Sette, Pittaco, di cui Diogene in I 81 riferisce: «Per lui, esercizio fisico era macinare il grano, come dice il filosofo Clearco" 27: analogamente, Clemente, Paed. III, 10, 50, 2 riporta: «II re Pittaco faceva girare la mola e cosi svolgeva un esercizio rinforzante». Ancora, a proposito di un altro dei Sette, Misone, il Laerzio cita Protag. 343 A: «Platone il filosofo, infatti, lo menziona anch'egli nel Protagora, ponendolo in luogo di Periandro" 28; cosi Clemente, Strom. I 14, 59, 5: "Platone mette Misone di Chene al posto di Periandro, indegno secondo lui del titolo di Sapiente, poiché fu tiranno".

23 Hdt. | 170, 3 dice solo che Talete era di origine fenicia (fr. 11 A 4 D.-K.; cfr. Suid. fr. 11 A 2 D.-K. e Schol. ad Plat. Resp. 600A = fr. 11 A 3 D.-K.; Duri., FGH 76 F 74; Democrit. fr. 68 B 115a D.-K.

${ }^{24}$ Cfr. Hippol. Refut. I 1, 3.

25 Per Plinio il Vecchio (Nat. Hist. II 53), Talete seppe prevedere l'eclissi del 585, del 28 maggio. II passo di Eudemo è il fr. 144 Wehrli; cfr. Eiusd. fr. 145 Wehrli, per cui Talete scopri l'eclissi del sole e il suo passaggio tra i tropici, che non si verifica sempre nello stesso modo. II passo di Senofane è il fr. $21 \mathrm{~B} 19 \mathrm{D}$. $\mathrm{K}$.; quello di Erodoto è 174, 2; quello di Eraclito, il fr. 22 B 38 D. -K. e quello di Democrito è il fr. 68 B 15a D.-K. Cfr. 11 A 5 D. ${ }^{-K}{ }^{26}$ Cfr. 9 A 1 D. K. FGH 2 T 11.

27 Fr. 71 Wehrli. Cfr. Plutarc. Conviv. Sept. Sap. 14, 157 e; Carmi popolari (PMG, fr. 23): «Macina, o mola, macina, / ché macina anche Pittaco, / sovrano della grande Mitilene".

28 Cfr. Pausan. $\times 24,1$. 
Un'espressione simile fin a livello lessicale e sintattico si trova sia in Diogene sia in Clemente a proposito della modalità grafica di un'opera di Anassagora: in 1111 Diogene attesta infatti: «E per primo Anassagora pubblicò anche un libro con una mappa», oìv $\gamma \rho \alpha \varphi \eta ฺ$ "con una cartina, una mappa, un disegno" ${ }^{29}$. Analogamente negli Strom. I 16, 78, 4 (= 59 A 36 D.K.) leggiamo: «riportano che Anassagora per primo abbia pubblicato un libro $\delta$ dà $\gamma \rho \alpha \varphi \tilde{r}$. Convergenze tanto strette non possono essere casuali e sembrano dovute o a una fonte comune o ad una conoscenza diretta.

In 1119 Diogene cita, a proposito di Socrate, una poesia: «Perciò, fra I'altro, Timone nei Silli dice ${ }^{30}$ : 'Dunque da questi deviò lo scultore che ciarlava di legalità, / dei Greci incantatore, che rendeva precisi nel parlare, / nasuto del naso di un retore, ironico mezzo attico'». La stessa poesia è citata, per i primi tre versi, da Clemente, Strom. I 14, 63, 3: «A lui succede Archelao, di cui fu scolaro Socrate: ma egli..." segue la citazione, "cosi dice Timone nei Silli, per il fatto che Socrate si volse dai problemi fisici a quelli etici». Sempre a proposito di Socrate, un'altra poesia, in Diog. L. II 25 (Filemone, fr. 105, 45 Kassel-Austin, PCG VII), trova riscontro in Clemente Alessandrino, Paedag. II 108, 4. $E$ in II 33 il seguente pensiero di Socrate: " $E$ diceva di meravigliarsi di quanti si fanno fare delle effigi di pietra, perché si preoccupano della pietra, che sia il più somigliante possibile, mentre non si curano di se stessi, di non aparire simili alla pietra", si avvicina a quello di Clemente, Protr. 62, $3^{31}$.

Di Aristippo, Diogene in II 75 ricorda una celebre battuta: «A quanti, dunque, lo biasimavano, ribatté: “lo posseggo Laide, ma non ne sono posseduto, poiché la cosa migliore è il dominare i piaceri e non lasciarsene vincere, non il non soddisfarli" "32, registrata anche da Clemente Alessandrino, Strom. II 20, 118, 3: "Aristippo di Cirene: quando lo si rimproverava di frequentare assiduamente la meretrice di Corinto, rispondeva: 'Sono io che possiedo Laide, non lei che possiede me's: Clemente presenta la posizione di Aristippo rispetto al piacere come analoga a quella dei Nicolaiti ${ }^{33}$. Ancora riguardo alla concezione del piacere di Aristippo e dei Cirenaici, che ammettevano solo il piacere cinetico, in contrasto con quella di Epicuro che prediligeva il piacere catastematico, troviamo una convergenza tra Diogene e Clemente: Diogene in II 89 ricorda:

\footnotetext{
${ }_{29}$ Congettura di H. Kochte, "Fleckeisen's Jahrbücher» 133 (1886), p. 769, accolta dall'editore Marcovich

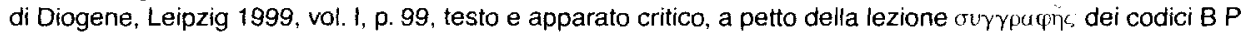

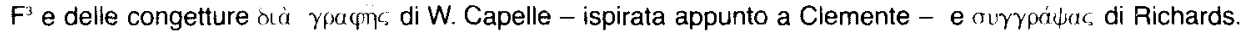

30 Timone, B 25 Diels; fr. 50 Wachsmuth = Supplementum Hellen. 799.

31 Sull'apprezzamento di Socrate nella Patristica cfr. F. BENZ, Christus und Sokrates in der alten Kirche, "Zeitschrift für die neutestamentliche Wissenschaft" 43 (1950-51), pp. 195-224.

32 Aristippo, fr. 57 A Mannebach; IV A 96 Giannantoni. Cfr. Cic. Epp. ad Famil. IX 26, 2; Gnomol. Vatic. 65.

33 Un altro parallelo con Diogene, sempre a proposito di Aristippo, occorre nel Paedagogus clementino, II 8,64, 1 e 69, 1, che somiglia a Diog. Laert. II 76: "A Caronda - secondo alcuni, invece, a Fedone - che domandò: "Chi è quello che si è cosparso di unguento?", "lo", rispose, "lo sventurato, e ancora più sventurato di me il re dei Persiani; ma vedi che, come nessuno degli altri animali si trova diminuito per questo in alcunché, cosi neppure un essere umano: malvagi come sono, malamente periscano i cinedi che screditano il nostro bell'unguento" (Aristippo, fr. 77 C Mannebach; IV A 63 Giannantoni; Sen. Ben. VII 25, 1.
} 
«Ma l'eliminazione del provare dolore -come è formulata da Epicuro- a loro sembra che non sia un piacere: neppure l'assenza di piacere sarebbe un dolore. Infatti, entrambi consistono nel movimento, mentre non è movimento l'assenza di pena, né l'assenza di piacere, poiché l'assenza di pena è condizione di quiete simile a quella di uno che stia dormendo" ${ }^{34}$. Similmente, gli Anniceriani, di ascendenza appunto cirenaica, confrontavano questa condizione epicurea del piacere catastematico con quella di un morto, come attesta Clemente, Strom. II 21, 130, 7-8: «I cosiddetti Anniceriani, provenienti dalla tradizione cirenaica, non fissarono nulla come fine determinato della vita in genere, ma sostennero che il fine è specifico per ogni singola azione, ed è il piacere che dall'azione stessa nasce. Questi Cirenaici respingono la definizione di piacere data da Epicuro, cioè l'eliminazione di ciò che procura dolore: essi lo chiamano lo stato di un cadavere". Ancora dottrina epicurea del piacere è oggetto di un altro stretto parallelismo tra Diogene, II 90: “(gli Epicurei)

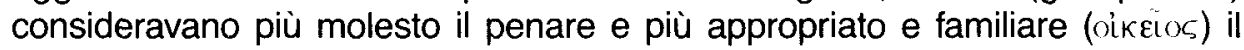
provare piacere", e Clemente, Strom. II 21, 128, 1, che ci riporta direttamente un frammento di Epicuro, il fr. 509 Usener: “Epicuro e i Cirenaici dicono che il primo impulso proprio (oíkદios) della natura è il piacere; la virtù, secondo loro, sarebbe subentrata in funzione del piacere e avrebbe ingenerato il piacere».

Se Epicuro, per la sua etica incentrata sul piacere, non è particolarmente amato da Clemente e dai Padri Cristiani in genere, Platone è indubbiamente considerato il massimo filosofo greco da Clemente, che lo loda grandemente fin dall'inizio degli Stromata ${ }^{35}$ e che ne riprende molti temi ${ }^{36}$. Anche Diogene accorda un posto di straordinario rilievo al filosofo ateniese nelle sue Vitae, dedicando un intero libro a lui, il III, e un altro agli Accademici, il IV; soltanto a Epicuro è accordato un onore simile di avere un intero libro per lui, il $X$, quantunque esso comprenda già anche gli Epicurei e non ci sia un altro libro dedicato ai seguaci di Epicuro. Alcuni parallelismi puntuali tra Diogene e Clemente sono i seguenti. In III 46, Diogene elenca i seguaci di Platone, tra cui «ci sono anche due donne, Lastenia di Mantinea e Assiotea di Fliunte, la

${ }^{34}$ Aristipp. fr. 204 Mannebach; Epicur. fr. 450 Usener: della racolta useneriana ho curato una traduzione con aggiornamenti e apparati: Epicurea, Milano 2002, cui rinvio per i commenti ad loc.

${ }_{35} \mathrm{Si}$ veda R. CASEY, Clemens and the Beginnings of Christian Platonism, "Harvard Theological Review» 18 (1925), pp. 39-101; J. MEIFORT, Platonismus bei Clemens Alexandrinus, Tübingen $1928 ; H$. MERKT, Homoiosis Theôi. Von der platonische Angleichung an Gott, Freiburg in S. 1952; E. OsBORN, The Philosophy of Clemens Alexandrinus, Cambridge 1954; J. WASzINK, Der Platonismus und die altchristliche Gedankenwelt, in Recherches sur la tradition platonicienne. Entretiens sur I'Antiquité Classique, Genève 1955, pp. 139-174; J. Wrtzes, The Twofold Way: Platonic Influences in the Work of Clemens, I: "Vigiliae Christianae" 11 (1957), pp. 226-245; Il: ibid, 14 (1960), pp. 129-153; S. LillA, Middle Platonism, Neoplatonism and Jewish-Alexandrine Philosophy, "Archivio Italiano per la Storia della Pietà 3 (1962), pp. 3.36; M. CHADWICK, Early Christian Thought and the Classical Tradition, Oxford 1966; J. WHITTAKER,

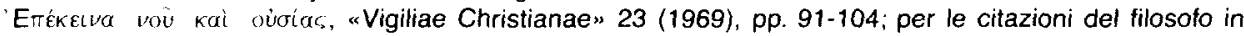
Clemente: F. CLARK, Citations of Plato in Clement of Alexandria, "Transactions and Proceedings of the American Philological Association" 33 (1902), pp.12-20.

36 Per citare un esempio fra i tanti, Clemente si riferisce alla concezione delia filosofia come meditatio mortis, espressa da Plat. Phaed. $67 \mathrm{E} ; 81 \mathrm{~A}$ etc., dicendo che nella Chiesa cristiana si trova inverata: «Piena è tutta la Chiesa di quelli che hanno meditato per l'intera vita la morte, scintilla di vita in Cristo: di uomini e anche di donne virtuose" (Strom. IV 8, 58, 2). 
quale si vestiva anche da uomo, come afferma Dicearco". Clemente, parlando delle donne sagge in Strom. IV 19, 122, 2, ricorda anch'egli che «con Platone studiavano filosofia Lastenia di Mantinea e Assiotea di Fliunte ${ }^{37}$. Soprattutto, Clemente Alessandrino, in tutto il passo di Strom. II 5, 20-24, trattando di come la dottrina greca sia improntata alla mosaica, porta gli esempi a suo avviso migliori dal più sapiente dei filosofi greci, Platone, che per lui, come abbiamo accennato, è emulo di Mosè.

Nel libro IV, dedicato agli Accademici, Diogene, parlando di Senocrate,

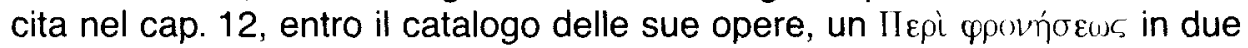
libri di cui proprio Clemente ci fornisce un frammento in Strom. II 5, 24, 1, mostrando l'accordo delle teorie di questo filosofo platonico con un passo biblico sapienziale: "Giustamente dunque è detto nel libro di Salomone (sc. Sir 31 [34], 8): 'La sapienza è sulla bocca degli uomini di fede'. $E$ in verità

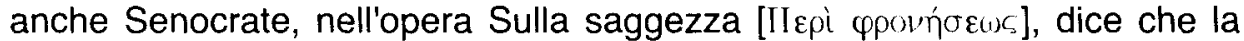
sapienza è conoscenza delle cause prime e dell'essenza intelligibile. Duplice egli ritiene la saggezza: saggezza pratica e saggezza teoretica; e questa sarebbe appunto la sapienza umana. Per cui la sapienza ( $\sigma o \phi i ́ a)$ è saggezza, ma non ogni saggezza è sapienza» ${ }^{38}$. Similmente, in un altro catalogo di opere, quelle di Teofrasto, Diogene ( $V 50)$ ne ricorda una, Achicaro, in un libro, sul titolo della quale ci illumina ancora Clemente in Strom. I 15, 69, 3-5: «Democrito ha fatto sue le dottrine morali dei Babilonesi. Si dice infatti che egli interpretò la stele di Akikaros e la inserì nei propri scritti; lo si può rilevare dalle sue parole: egli scrive: 'Questo dice Democrito'. In particolare dice di sé in un passo, vantandosi della sua cultura: 'lo girai per il mondo... conobbi innumerevoli sapienti...'. E percorse infatti Babilonia, Persia, Egitto, sempre alla scuola dei Magi e dei sacerdotis ${ }^{39}$. E evidente che il passo clementino si inserisce sempre nel discorso dell'antichità e dell'importanza delle dottrine barbariche rispetto a quelle greche. Inoltre, una massima di un discepolo di Teofrasto, Demetrio Falereo, è presente sia a Clemente Alessandrino, Paed. III 33, 3, sia a Diogene Laerzio, V 82: «I giovani, disse che a casa devono avere rispetto per i genitori, per strada devono averlo per quelli che incontrano e, in solitudine, per se stessi»40.

In apertura del VI libro delle Vitae philosophorum, dedicato ai Cinici, Diogene, nel cap. 1, incomincia a presentare Antistene e la sua ascendenza, secondo alcuni ateniese, secondo altri frigia: «Antistene, figlio di Antistene,

${ }_{37}$ Entrambe le donne figurano anche nella lista delle discepole di Speusippo (Diog. Laërt. IV 2); cfr. Anonimo, Prolegom. In Plat. 4; Athen. XII 546 D; Themist. 23, 295 C. Si veda T. Dorandi, Assiotea e Lastenia: due donne all'Accademia, "Atti e Memorie dell'Accademia Toscana La Colombaria" 54 (1989), pp. 51-66.

${ }^{38}$ Senocrat. fr. 6 Heinze $=259$ Isnardi Parente, Senocrate, Napoli 1982, pp. 254 e 427-429.

39 Secondo G. Wissowa (in P.-W. I 1, 1893, col. 1168) e H. Diels (in D.-K. II p. 208) il personaggio citato da Teofrasto sarebbe appunto il babilonese Achikâr, la cui stele fu tradotta da Democrito secondo Clemente. Anche il libro veterotestamentario di Tobia, 1, 22-22, presenta Achîkâr come il giusto perseguitato e poi ricompensato. Si veda F. NAU, Histoire et sagesse d'Ahikar, Paris 1909; per il libro di Achikar A.M. DENIS, Introduction aux pseudépigraphes grecs d'AT, Leiden 1970, pp. 201-214.

40 Demetr. fr. 117 Wehrli. 
ateniese. Si diceva, tuttavia, che non fosse un indigeno. Perciò, a chi lo criticava avrebbe detto: 'Anche la madre degli dèi è una che viene dalla Frigia' ${ }^{141}$. Aveva, infatti, fama di essere figlio di madre tracia: perciò anche a Tanagra, essendosi guadagnato gloria in battaglia, a Socrate diede àdito a dire che da due Ateniesi non sarebbe nato cosi nobile ${ }^{42}$. Ed egli stesso, esprimendo il proprio disprezzo verso gli Ateniesi che si vantavano di essere 'nati dalla terra', soleva affermare che essi non erano per nulla di stirpe più nobile rispetto alle chioccioline e alle cavallette ${ }^{43}$. Naturalmente, la teoria delle origini frigie di Antistene è accolta da Clemente come esempio della derivazione dai barbari della filosofia greca, in Strom. I 14, 66, 1-2: «Questa è dunque l'epoca dei più antichi sapienti e filosofi greci. E c'è forse bisogno di aggiungere che la maggior parte di loro erano di stirpe barbara, ed educati presso barbari, se è vero che Pitagora era presentato come etrusco o tirio, Antistene era un frigio e Orfeo odrisio o trace? I più dichiarano addirittura Omero un egiziano!». Di Antistene, in VI 3, il Laerzio ricorda una recisa dichiarazione antiedonistica: «E soleva dire, continuamente: 'Possa io essere pazzo, piuttosto che provare piacere' ${ }^{\prime}{ }^{44}$, che Clemente riporta, negli identici termini di Diogene Laerzio, entro una serie di esempi di come anche molti filosofi greci abbiano ripudiato il piacere, in Strom. II 20, 121, 1: "Antistene preferisce essere pazzo, piuttosto che provare piacere».

Piena convergenza si trova nel Laerzio e in Clemente anche riguardo a un aneddoto relativo a Diogene il Cinico, che il dossografo narra come segue in Vit. VI 39: «Siccome un eunuco malvagio aveva fatto scrivere sulla porta di casa: 'Non entri nulla di male', osservò: 'E il padrone di casa, allora, per dove potrebbe entrare?'»45. Clemente, in Strom. VII 4, 26, 1, riferisce nei medesimi termini: «Diogene aveva trovato scritto sulla casa di un ribaldo: 'Qui abita Eracle vittorioso: nessun vizio entri'; e commentò, con molto spirito: 'E come farà ad entrare il padrone di casa?'». Sempre su Diogene il Cinico, il Laerzio in VI 60 ricorda che, "Siccome Frine aveva posto a Delfi, come offerta votiva, una statua d'oro di Afrodite, si dice che egli vi abbia scritto sopra: 'Da parte dell'intemperanza della Grecia'» ${ }^{46}$, un aneddoto noto anche a Clemente, Paed. III 21, $4^{47}$.

${ }^{41}$ Cfr. Plut. De exil. 607AB; Sen. De const. sap., 18, 5; F. Decleva Caizzi, Antisthenis Fragmenta, Milano 1966, nrr. 122-124 e p. 118.

42 Cfr. Antisthen. frr. 1 e 3 Giannantoni, SSR V A.

43 Antisthen. fr. 8 Giannantoni.

${ }^{44}$ Antisthen. fr. 122 Giannantoni; Sext. Emp. Pyrrh. Hypot. III 181; Adv. math. XI 73-74

45 Diog. fr. 347 Giannantoni. Lo stesso aneddoto è riferito due volte da Diogene Laerzio, che probabilmente lo riprende da due fonti diverse; in VI 50 troviamo infatti: "Siccome un novello sposo aveva apposto davanti a casa la seguente iscrizione: 'll figlio di Zeus, Eracle, dalla bella vittoria, / qui allinterno dimora: non entri male alcuno', egli vi aggiunse: 'Dopo la guerra, I'alleanza'».

46 Diog. fr. 212 Giannantoni; cfr. Schol. in Aristoph. Plut. 179

47 Del cinico Cratete di Tebe in VI 85 Diogene riferisce che «Di lui si tramandano i seguenti Versi di trastullo: 'Una città esiste, Pera, entro fumo colore del vino, / bella e opulenta, e sporca ovunque, e non possiede niente, / non naviga in essa nessun uomo stolto, né un parassita, / né un crapulone, che gode del retro di una cortigiana; / bensi essa produce timo e aglio e fichi e pani, / perciò, gli abitanti per questi non si fanno guerra reciproca: / essi non prendono armi per soldi, né a scopo di gloria' (Anthol. Palat. Append. $V$ 13 Cougny; PPF 10 B 4; Supplementum Hellen. 351; Cratet. fr. 70 Giannantoni; Demetr. Elocut. 259; Apul. Apol. 22): si veda il parallelo con Clemente, in Paed. II 93, 4. 
I punti di contatto tra Diogene e Clemente si fanno particolarmente numerosi e importanti riguardo a Pitagora, a cui il Laerzio dedica la prima parte del libro VIII48 delle Vitae in qualità di iniziatore della scuola italiota - come lo è per Clemente, Strom. I 14, 62, 1: «la scuola filosofica italica, derivante da Pitagora» --: già in apertura del libro stesso, trattando delle origini di Pitagora, Diogene concorda con Clemente: «Pitagora, figlio di Mnesarco, incisore di pietre preziose per anelli, di Samo, come dice Ermippo, oppure, come afferma Aristosseno, tirreno, originario di una di quelle isole che gli Ateniesi presero in possesso, dopo averne scacciati i Tirreni. Alcuni, poi, sostengono che egli fosse figlio di Marmaco figlio di Ippaso figlio di Eutifrone figlio di Cleonimo, esiliato da Fliunte, e che Marmaco abitasse a Samo, motivo per cui Pitagora si dice originario di Samo" (Diog. Laërt. VIII 1) ${ }^{49}$. Analoghe connessioni con la Fenicia e la Tirrenia/Etruria per Pitagora sono poste in luce da Clemente in Strom. 114, 62, 2: sempre nel contesto delle origini barbare della filosofia greca: «Pitagora, dunque, figlio di Mnesarco, era di Samo, come dice Ippoboto; come, invece, affermano Aristosseno nella Vita di Pitagora ed Aristarco e Teopompo, era un Tírreno; secondo Nearco, invece, era originario della Siria o di Tiro, cosicché, per la stragrande maggioranza degli studiosi, Pitagora era barbaro di stirpe»; ibid. 15, 66, 1: «Pitagora era presentato come Tirreno o Tirio ${ }^{50}$. Anche i presunti soggiorni egiziano e mesopotamico di Pitagora sono ricordati sia da Diogene sia da Clemente. II Laerzio in VIII 3 riferisce infatti: "In particolare, fu in Egitto, dove Policrate lo raccomandò ad Amasi per lettera; e imparò la lingua di questa gente, secondo quanto afferma Antifonte nell'opera Su coloro che hanno primeggiato in fatto di virtu. $E$ fu anche presso i Caldei e presso i Magi. Quindi, a Creta discese con Epimenide nell'antro dell'Ida; ma anche in Egitto entrò nei recessi dei santuari, e apprese le dottrine relative agli dèi in segreto "51. Analogamente, l'Alessandrino, in Strom. I 15, 66, 2 insiste su questo apprendistato di Pitagora presso i barbari: «Di Talete s'è detto che era fenicio e che ebbe rapporti con i profeti egiziani, come pure Pitagora: li frequentò, e per essi anzi si circoncise, per poter adire ai sacrari dei templi e apprendervi dagli Egiziani la filosofia mistica. Egli convisse poi con i più celebri fra i Caldei e i Magi, e il suo termine homakoeion adombra quello che noi oggi chiamiamo 'chiesa'». Anche una notizia di lone di Chio relativa a una presunta opera di Pitagora è comune a

48 Abbastanza curiosamente, per il libro VII di Diogene Laerzio, non abbiamo, a quanto sembra, parallelismi specifici con Clemente, il quale pure apprezzava gli Stoici soprattutto in campo etico e ne assimitò il pensiero (come mostrano J. Stenzelberger, Die Beziehung der frühchristlichen Sittenlenre zur Ethik der Stoa, München 1933; M. Spanneut, Le Stoïcisme des Pères, Paris 1957; W. Richardson, The Basis of Ethics: Clement and Chrysipp, "Studia Patristica" IX, 1963, pp. 87-97; D. BRADLEY, The Transformation of Stoic Ethics in Clement Alexandrinus, "Augustinianum» 14, 1974, pp. 41-66) al punto da attingere a piene mani, nel Paedagogus e negli stessi Stromata, alle Dissertationes del neostoico Musonio, redatte da un discepolo: cfr. J.M. BLAZOUEZ, "El uso del pensamiento de la filosofia griega en el Pedagogo de Clemente de Alejandria", Anuario de Historia de la lglesia, 3 (1994), p. 59 sgg.; I. RAMElu, 'Dio come padre nello Stoicismo romano al tempo della predicazione cristiana e nell'“Epistola Anne'", in Scripta antigua in honorem A. Montenegro Duque-J.M. ${ }^{2}$ Martínez, edd. S Crespo Ortiz de Zárate-A. Alonso Ávila, Valladolid, 2002, pp. 343-351 (si vedano i casi più importanti segnalati nella mia edizione di Musonio Rufo, Diatribe, frammenti, testimonianze, con testo a fronte, introduzione e note, Milano 2001).

49 Hermipp. fr. 19 Wehrli; Aristox. fr. 11a Wehrli.

50 Porfirio, in Vita Pythag. 1, sostiene che Pitagora fu istruito dai Caldei in Fenicia e che, al suo ritorno a Samo, segui le lezioni di Ferecide di Siro.

51 Cfr. lambl. Vita Pythag. $18 \mathrm{sgg}$. 
Diogene, VIII 8 («lone di Chio, poi, nelle sue Triadi, afferma che egli ascrisse alcuni suoi componimenti a Orfeo»52) e a Clemente, Strom. I 21, 131, 4: «lone di Chio nelle Triadi riporta che anche Pitagora attribui ad Orfeo alcuni suoi scritti”. Similmente, anche un motto della moglie di Pitagora, Teanò, è riportato in modo identico sia da Diogene sia da Clemente. II primo, in VIII 43, scrive: «E riferiscono pure che, quando le [sc. a Teanò] fu domandato dopo quanti giorni una donna si purifichi dal contatto intimo con un uomo, ella rispose: 'Dal contatto con il proprio sposo, immediatamente; da quello con un estraneo, mai'53. E Clemente Alessandrino, Strom. IV 19, 121, 3: «E che dire della pitagorica Teanò? Non giunse forse a grande altezza filosofica?... Della stessa austerità di costumi si riferisce a prova anche il seguente motto: le fu domandato dopo quanto tempo una donna levatasi dal connubio con un uomo potesse recarsi alle feste delle Tesmoforie; rispose: 'Se dal connubio con il proprio marito, anche subito; se da quello con un estraneo, mai'».

Diogene Laerzio, in VIII 59, trattando di Empedocle, cita una sua poesia: "'E, quanti filtri ci sono a scudo di mali e vecchiaia, / tu imparerai, poiché io tutto questo per te solo eseguo. / L'impeto farai cessare di venti indomabili, i quali / si alzano sopra la terra e coi soffi rovinano i campi; / e, se vorrai, poi di nuovo richiamerai $\mathrm{i}$ loro spiri. / $\mathrm{E}$, dopo la nera pioggia, farai venire per gli uomini / una opportuna secchezza, e poi dal secco d'estate / farai venire acquazzoni che alimentano gli alberi, / e scenderanno dall'etere, e condurrai, poi, dall'Ade / l'anima di un uomo morto ${ }^{154}$. Afferma, poi, Timeo nel diciottesimo libro che il nostro uomo è stato ammirato sotto molti rispetti. $E$ infatti, quando una volta $i$ venti etesii presero a soffiare troppo violentemente, in modo da rovinare le messi, ordinò di scuoiare degli asini e fece disporre le loro pelli sulle colline e sulle alture, allo scopo di catturare il vento: e, siccome questo cessò, fu chiamato 'Impeditore del Vento'". Alla stessa poesia e allo stesso episodio si riferisce Clemente, Strom. VI 3, 30, 2: “Empedocle di Agrigento fu soprannominato 'Impeditore del Vento'. Si racconta che dalle montagne di Agrigento si era messo a spirare un vento pericoloso e pestilenziale per gli abitanti, e che per di più provocava sterilità nelle loro donne. Empedocle lo fece cessare. Perciò scrive egli stesso nelle sue poesie: 'L'impeto farai cessare di venti indomabili, i quali / si alzano sopra la terra e coi soffi rovinano i campi; / e, se vorrai, poi di nuovo richiamerai i loro spiri'. E invitava a seguirlo 'quelli che sono bisognosi di previsioni e quelli che sono da lungo tempo trafitti da dolorosi morbi'». Queste ultime parole rielaborano l'ultima parte di un'altra poesia empedoclea citata dal Laerzio in VIII 61: "O amici, che abitate la città grande sopra il biondo / Agrigento ... salve! lo passo tra voi quale dio immortale... / quando entro con questi in città prosperose, / siano uomini o donne, mi si venera: e mi tengono dietro / decine di migliaia, per sapere quale sia la via al profitto: / alcuni avevano consultato oracoli, altri per malattie di ogni

52 FGH 392 F 25a; 36 B 2 D. K.

53 Cfr. Stob. Anth. IV 23, 53.

54 31 B 111 D. - K. = fr. 101 Wright; cfr. Suda, A 3242; Tzetz. Chiliad. II 906-914 = 909-917 Leone. 
genere / volevano udire una voce acutissima» 55 . Un'altra poesia di Empedocle è citata sia da Diogene sia da Clemente: in Vitae, VIII 77 il Laerzio riporta infatti: «E a suo parere l'anima riveste ogni sorta di forma di animali e di piante. Dice in particolare: 'Già, infatti, un tempo io fui fanciullo e anche fanciulla, / cespuglio, uccello, pesce che balza fuori del mare, infiammato's ${ }^{56}$. Clemente Alessandrino, Strom. VI 2, 24, 3, cita analogamente: «il celebre passo di Empedocle, 'Già, infatti, un tempo io fui fanciullo e anche fanciulla, / cespuglio, uccello, pesce muto nel mare', è parafrasato da Euripide nel Crisippo...».

Anche su Eraclito si possono trovare cospicui paralleli tra i due autori: al principio del libro IX, dedicato ai filosofi "sporadici» e agli Scettici, il Laerzio scrive: «(Eraclito) ebbe elevato sentire, più di chiunque altro, e fu altero, come risulta chiaro anche dalla sua opera scritta, in cui dice: 'L'erudizione, per quanto molta e varia, non educa l'intelletto: altrimenti, infatti, lo avrebbe educato a Esiodo, a Pitagora e, ancora, a Senofane e a Ecateo' ${ }^{157}$. E infatti 'una sola la sapienza: sapere pensare, in quanto il pensiero guida tutto dovunque' ${ }^{\prime}{ }^{58}$. Anche Clemente sa che Eraclito nominava Pitagora, e ne fa un argomento in favore della priorità cronologica di quest'ultimo in Strom. I 21, 129, 4, in un discorso cronologico simile a quello svolto negli ultimi capitoli dell'Oratio ad Graecos di Taziano: «Eraclito poi è posteriore a Pitagora, poiché lo menziona nel suo libro" 59 .

Un passo poetico parmenideo è citato sia dal Laerzio in IX 21 sia da Clemente. Diogene riporta: «È necessario che tu tutto sappia, / sia il cuore ben saldo di Verità che persuade, / sia le opinioni dei mortali, in cui non c'è verace garanzia» ${ }^{60}$; cosi Clemente, Strom. $\vee 10,59,6:$ : $\mathrm{il}$ grande Eleate, Parmenide, introduce la dottrina delle due vie scrivendo così: 'sia il cuore ben saldo di Verità che persuade, / sia le opinioni dei mortali, in cui non c'è verace garanzia'". Anche l'eroica morte del parmenideo Zenone di Elea in Diogene (IX 27) per opera di un tiranno rientra nella topica citata pure da Clemente. Diogene scrive: « $E$, poiché volle rovesciare il tiranno Nearco, fu arrestato, secondo quanto dice Eraclide nell'epitome di Satiro ${ }^{61}$ : in tale occasione fu anche interrogato, perché facesse i nomi dei complici e desse spiegazioni delle armi che portava a Lipara, ed egli fece il nome di tutti gli amici di lui, volendo lasciarlo isolato. Poi, sostenendo di avere da parlargli all'orecchio a proposito dei certe questioni, lo morsicò lì e non lo lasciò

5s 31 B 112 D =fr. 1 Zuntz = fr. 2 Wright. Anthol Palat. IX 569; Anthol. Planud.; Sext. Emp. Adv. Mathem. 1302 e passim. Heraclid. fr. 77 Wehrli.

5631 B 117 D.-K. = fr. 34 Zuntz = fr. 108 Wright. Cfr. Athen. VIII 365 E; Hippol. Refut. I 3, 2; Anthol. Palat. IX 569, 1-2 et alibi.

57 Heraclit. B 40 D.-K.; fr. 16 Marcovich; cfr. il mio Allegoria, I, L'età classica, in collab. con G. Luccetta, Milano 2004, cap. 1.

58 Heraclit. B 41 D.-K. = 85 Marcovich.

59 Sul rapporto tra Eraclito e Clemente, che passa attraverso la dottrina del logos, si veda P. VALENTIN, Héraclite et Clément, "Recherches de Sciences Religieuses" 46 (1958), pp. 27-58.

60 Parmenid. B 1, 28-30 D.-K. I versi sono citati da Sesto Empirico (Adv. math. VII 111 e 114), Procio (in Platon. Tim. 1. p. 345, 15 Diehi) e Simplicio (in Aristot. De cael. p. 557, 25 Heiberg).

64 Heraclid. Lemb. fr. 7, FGH III 169. 
andare finché non fu ferito a morte, subendo una fine analoga a quella del tirannicida Aristogitone ${ }^{62}$. Demetrio, invece, negli Omonimi afferma che gli tranciò piuttosto il naso ${ }^{63}$. Antistene, poi, nelle Successioni dei filosofi, afferma che a lui, dopo che ebbe denunciato gli amici del tiranno, fu domandato da quest'ultimo se ci fosse qualcun altro: ed egli rispose: 'Tu, la sciagura della città'. E si rivolse agli astanti: 'Mi meraviglio della vostra viltà, se siete schiavi del tiranno a causa (del timore) di quanto io sto sopportando'. E, tranciatasi la lingua, la sputò verso di lui: e i suoi concittadini, infiammati, subito lapidarono il tiranno"64. Così Clemente Alessandrino, nella topica dei saggi perseguitati dai tiranni di Strom. IV 8, 57: («anche se sono uccisi dalle torture dei tiranni, restano appesi all'amicizia divina"), in IV 8, 56, ricorda lo stesso episodio: "Zenone di Elea, quando lo si volle costringere a rivelare un segreto, resistette ai tormenti senza confessare nulla, e alla fine si mozzò la lingua e la sputò in faccia al tiranno - c'è chi lo chiama Nearco, chi Demilo".

A proposito delle predizioni del futuro da parte di Democrito e del suo debito verso il fratello Damaso, si può riscontrare un'evidente somiglianza tra Diogene IX 39 («che egli, ritornato dal suo viaggio all'estero, trascorreva la vita nelia più profonda povertà, dato che aveva dilapidato tutto il suo patrimonio, e che, per la sua mancanza di mezzi, era mantenuto da suo fratello Damaso. Ma, siccome riusci a predire eventi futuri65, prese a godere di buona fama") e Clemente Alessandrino, Strom. VI 3, 32, 2: «Democrito, che fece molte previsioni in base alle osservazioni dei fenomeni celesti, fu soprannominato 'Sapienza'. Lo aveva accolto benevolmente il fratello Damaso, ed egli, congetturando dalla posizione di certi astri, predisse che ci sarebbe stata una gran pioggia: quelli che gli credettero raccolsero al riparo le messi... gli altri perdettero tutto". In IX 50, inoltre, anche Diogene ricorda il soprannome di Democrito: «Protagora, per altro, ascoltò le lezioni di Democrito. E (Democrito) era chiamato "Sapienza»66. Anche Clemente, Strom. I 14, 64, 4 mostra di sapere che Protagora era discepolo di Democrito: «Discepoli di Democrito furono Protagora di Abdera e Metrodoro di Chio, del quale fu discepolo Diogene di Smirne, di cui fu allievo Anassarco; e di quest'ultimo lo fu Pirrone, del quale lo fu Nausifane ${ }^{67}$. Che Anassarco fosse discepolo di Diogene, a sua volta allievo di Metrodoro, allievo di Democrito, è noto anche al Laerzio, IX 58: «Anassarco di Abdera. Costui ascoltò le lezioni di Diogene di Smirne, e quest'ultimo quelle di Metrodoro di Chio; Metrodoro, a sua volta, sarebbe stato discepolo... di Democrito". Proprio riguardo ad Anassarco, e in particolare alla sua morte, troviamo un parallelismo molto stretto fra Diogene, IX 59, e Clemente, Strom. IV 8, 56, 4, nel contesto già visto delle persecuzioni

62 Cfr. Diod. Bibl. hist. $X$ 18, $2=29$ A 6 D.-K.; Aristot. Const. Athen. 18, 4; Sen. De ira, II 23.

63 Demetr. Magnet. F 28 Mejer.

${ }_{64}$ Antisth. Rod. FGH 508 F 11. Sull'eroica morte di Zenone cfr. Plut. Adv. Colot. 1126 D; De Stoic repugn. $1051 \mathrm{C}$.

65 Cfr. Plin. Nat. Hist. XVIII 273 e 341

66 Cfr. Suid. S.v. Democrito, $\triangle 447$; Plat. Protag. 316 A 1.

${ }_{67}$ Cfr. Eus. Praep. Evang. XIV 17, 10. Per Anassarco: 72 A 1 D.-K. (fr. 1 Dorandi). 
dei sapienti per opera dei tiranni. II Laerzio scrive infatti: «Ebbe come nemico Nicocreonte, tiranno di Cipro. E una volta, durante un simposio, quando Alessandro gli domandò che impressione avesse del banchetto, rispose: 'O re, tutto è sontuoso: rimarrebbe soltanto che fosse servita a tavola la testa di un satrapo', lanciando una frecciata a Nicocreonte. E quello, memore dell'offesa, dopo la morte del re, quando Anassarco, durante un viaggio in mare, dovette approdare a Cipro contro la sua volontà, lo fece catturare e gettare in un mortaio, quindi diede ordine che fosse battuto con pestelli di ferro. Ma egli, senza preoccuparsi della pena, pronunciò quel detto, che ha incontrato ampia fama: 'Schiaccia, schiaccia il sacco di Anassarco, ma Anassarco tu non lo colpisci!'. E, quando Nicocreonte diede ordine che gli fosse tagliata anche la lingua, si dice che egli se la sia tranciata via con un morso e che l'abbia sputata verso di luis ${ }^{68}$. Clemente ricorda in senso paradigmatico lo stesso episodio: «Taccio poi del fatto di Anassarco: mentre era percosso con pestelli di ferro dal tiranno, gli gridava: 'Pesta pure il sacco di Anassarco: tanto Anassarco non lo pesti!'».

Diogene Laerzio, in IX 115, nel capitolo relativo a Timone di Fliunte, parla del discepolo di lui «Praulo di Troade, il quale fu così costante d'animo, secondo quanto riferisce Filarco narrando la storia, da sapere resistere, benché condannato ingiustamente a morte per tradimento, disdegnando di rivolgere ai cittadini una sola parola» ${ }^{69}$. Forse è lo stesso di cui parla Clemente Alessandrino, Strom. IV 56, 2 anche se i codici danno la forma «Paolo» (IIavios, e non II paud(s): «Lo stesso (sc. la resistenza al tiranno) fecero il pitagorico Teodoto e Praulo, scolaro di Lacide, secondo i racconti di Timoteo di Pergamo nel libro Sulla intrepidezza dei filosofi e di Acaico nei Discorsi di etica".

Inoltre, alle "dottrine occulte" epicuree alludono tanto Clemente quanto Diogene quanto Clemente, in termini simili. II Laerzio, in $\times 5$, informa che Epicuro «avrebbe elogiato e adulato per questo stesso motivo anche Idomeneo, Erodoto e Timocrate, che resero note e chiare le sue dottrine occulte " 70 : il termine è kpúpl $a$, che ricorre identico anche in Clemente, Strom. $V 9,58,1$, nel contesto di una serie di esempi di dottrine esoteriche ${ }^{71}$ presenti nelle varie scuole filosofiche: «Non solo i Pitagorici e Platone si esprimevano per lo più per enigmi, ma anche gli Epicurei dicono di avere, lasciati dal maestro, degli scritti segreti, e di non permettere a tutti di consultare questi scritti». E anche riguardo all'espressione "per enigmi» di Platone e dei Pitagorici, Diogene a sua volta fa un cenno: in III 63 dice infatti di Platone che "Si è poi servito di termini variegati perché la sua trattazione risultasse non facilmente comprensibile ai non esperti»; quanto ai Pitagorici, nel libro VIII tratta ampiamente della segretezza delle loro dottrine al cap. 15 ( «Fino al tempo di Filolao non fu possibile conoscere nessuna dottrina pitagorica:

68 Cfr. Diog. Laërt. IX 27; Athen. VI 250 F-251 A; Plut. Alex. 28, 4; Dio Chrys. 37, 45; Val. Max. III 3 ext. 4; Cic. Nat. deor. III 82; Plin. Nat. Hist. VII 87

69 Philarch. FGH 81 F 67.

70 Usener, fr. 124.

"Si veda E. FORTiN, Clement and the Exoteric Tradition, "Studia Patristica» IX, 3, Berlin 1966, pp. 41-56. 
questi soltanto pubblicò quei tre famosi libri per comperare i quali Platone mandò cento mine ${ }^{72}$. E più di seicento persone andavano da lui per ascoltarne le lezioni serali. E, se alcuni per caso avevano l'onore di poterlo vedere, scrivevano ai conoscenti che era toccata loro una grande fortuna. Gli abitanti di Metaponto, in verità, solevano chiamare la sua casa "tempio di Demetra", e la sua strada, <poi>, "museo", come ricorda Favorino nelle sue Storie miscellanee ${ }^{73}$. Anche gli altri Pitagorici erano soliti dire che non tutte le dottrine andavano rivelate a tutti, come afferma Aristosseno nel decimo libro delle Norme dell'educazione " ${ }^{74}$ ) e dei loro precetti simbolici al cap. 17 («i suoi precetti allegorici erano i seguenti: non attizzare il fuoco con un pugnale, non passare sopra la bilancia, non sedere sul chenice, non mangiare il cuore, non aiutare qualcuno a deporre il carico, bensi a sollevarlo ${ }^{75}$; avere le coperte sempre fatte su insieme, non portare in giro l'immagine di Dio su una pietra di anello, scompigliare l'impronta della pentola nella cenere, non astergersi con una piccola torcia per una seduta, non orinare rivolto verso il sole, non camminare sulla strada frequentata dal popolo ${ }^{76}$; non dare facilmente la destra, non tenere rondini sotto il proprio tetto, non allevare animali dagli artigli adunchi, non orinare né stare in piedi sopra le unghie e i capelli tagliati, volgere dall'altra parte il pugnale affilato, non voltarsi indietro quando si è ai confini, mentre si sta andando via") ${ }^{77}$, fornendone le spiegazioni nel cap. $18^{78}$, e ricordando poi anche altre prescrizioni pitagoriche di carattere enigmatico nei capp. 19-20 e 34-35, con le rispettive spiegazioni. La questione del simbolismo, per altro, fornisce un ulteriore punto di contatto fra la tradizione greca e Clemente: anche se non è questa la sede per trattare di un problema cosi complesso, è doveroso ricordare che l'Alessandrino recupera la tradizione dell'esegesi etimologico-allegorica ${ }^{79}$ che i Greci, soprattutto Stoici,

${ }^{72}$ Cfr. Diog. Laërt. VIII 6 e III 9.

73 Fr. 41 Mensching; cfr. Porphyr. Vita Pythag. 4: egli cita come fonte lo storico Timeo di Tauromenio (l'odierna Taormina), che forse è anche la fonte usata da Favorino.

74 Aristoxen. fr. 43 Wehrli.

${ }^{75}$ Cfr. lamblich. Vita Pythag. 84; Porphyr. Vita Pythag. 42 e passim.

${ }^{76}$ Cfr. Hesiod. Op. 727

77 Sui precetti simbolici pitagorici cfr. anche H. Saffrey, Une collection méconnue de symboles pythag. oriciens, "Revue des Études Grecques» 80 (1967), pp. 198-201.

78 «E, per lui, il non attizzare il fuoco con un pugnale significava non stuzzicare l'ira dei sovrani assoluti e la loro orgoglosa animosità. E il non trapassare la bilancia equivale non oltrepassare i confini dell'equità e della giustizia. Non sedersi sul chenice, poi, vuole dire avere pari cura del presente e del futuro: infatti, il chenice è la misura di cibo quotidiana. Con il precetto non mangiare il proprio cuore voleva indicare, poi, di non struggersi l'anima nelle pene e nei tormenti. Con il consiglio di non voltarsi indietro quando si è ai confini, mentre si sta andando via, voleva esortare quanti se ne stanno andando dalla vita a non mantenere un atteggiamento bramoso di vivere $e$ a non lasciarsi trascinare dai piaceri di questo mondo. E secondo gli stessi criteri è possibile comprendere anche i rimanenti precetti, per non attardarci troppo. [Cfr. Suda, II 3124; Giamblico, Protrettico, 21; Vita pitagorica, 82-86 (= 58 C 4 D.-K.); Portirio, Vita di Pitagora, 42 e passim; cfr. F. BoemM, De symbolis Pythagoreis, diss. Berolini 1905; A. DelATtE, Études sur la littérature pythagoricienne, Paris 1915; BURKERT, Weisheit, p. 150 sgg.]"

${ }_{79}$ Mi limito a ricordare U. Treu, Etymologie und Allegorie bei Klemens, "Studia Patristica» IV, 2 Berlin 1961, pp. 199-211, ma la bibliografia sarebbe molto ampia ed è abbondantemente fornita nel mio Allegoria, I, cit., dove offro anche una trattazione completa del tema. Per I'mportanza delia tematica in ambiente alessandrino cfr. D. Dawson, Allegorical Readers and Cultural Revision in Ancitn Alexandria, Berkeley-Los Angeles-Londion 1992. 
avevano applicato alla propria teologia espressa in forma mitica, trasferendola in campo cristiano; un'operazione culturale parallela era stata svolta da Filone di Alessandria in àmbito giudaico, che applicò all'Antico Testamento il metodo esegetico allegorico ${ }^{80}$.

Un intero passo della Epistula ad Menoeceum, conservataci da Diogene Laerzio, quello iniziale che costituisce un protrettico alla filosofia (Vit. phil. $X$ 122), è riportato alla lettera anche negli Stromata (IV 8, 69, 2-4): “Né uno, se è giovane, esiti a studiare filosofia, né, se si trova a essere vecchio, si stanchi di praticarla. Per nessuno, infatti, non è ancora il momento o non è più il momento di perseguire la salute dell'anima. Perché, chi dice che non è ancora il momento opportuno per la filosofia, o che questo momento è ormai passato, assomiglia a chi affermasse che non è ancora giunto il momento opportuno per la felicità, o che non lo è più. Cosicché, deve occuparsi di filosofia sia un giovane sia un vecchio, il primo perché, invecchiando, possa essere giovane nei beni, in grazia delle cose che sono state, l'altro per essere, al contempo, giovane e anziano, in virtù della mancanza di paura di quanto deve avvenire in futuro ${ }^{81}$.

Come si vede, i parallelismi finora indicati tra il Laerzio e l'Alessandrino sono molto cospicui: talora limitati a un aneddoto e a poche parole, talaltra si estendono maggiormente o si addentrano più a fondo nel pensiero filosofico di un autore o di una scuola. $\grave{E}$ vero che spesso sarebbe possibile spiegarli con una fonte dossografica comune - è stato fatto più volte il nome di Ippoboto- ${ }^{82}$; tuttavia è importante osservare che i punti enucleati rientrano anche, sovente, in un dibattito vivo all'epoca dei due autori, forse entrambi

80 Mi limito a rinviare, con bibliografia, a V. Nikiprowetzky, L'exégèse de Philon d'Aléxandrie, "Revue d'Histoire et de la Philosophie Religieuse" 53 (1973), pp. 309-329; D. Dawson, Allegorical Readers and Cultural Revision in Ancient Alexandria, Berkeley - Los Angeles - Oxford 1992; J.M. Dillon, The Formal Structure of Philo's Allegorical Exegesis, in AA.VV., Jacob Bernais. Un philologue juif, éd. J. Glücke - A Laks, Villeneuve d'Asq 1996, Cahiers de Philologie 16, pp. 123-131; P. Graffigna, Physikôs allegoreîn. Osservazioni lessicali sulla teoria allegorica filoniana, "KOINWNIA» 20 (1996), pp. 107-113; P. Borgen, Philo of Alexandria an Exegete for His Time, Leiden - New York - Köln 1997, Supplements to Novum Testamentum 86; A.A. LoNG, Allegory in Philo and Etymology in Stoicism: a Plea for Drawing Distinctions, in AA.VV., Wisdom and Logos. Studies in Jewish Thought in Honor of David Winston, edd. D.T. Runia - G.E. Sterling, Atlanta 1997. The Studia Philonica Annual 9, pp. 198-210; R. RADICE, Allegoria e paradigmi etici in Filone di Alessandria. Commentario al "Legum Allegoriae", pref. di C. Kraus Reggiani, Milano 2000, Temi metafisici e problemi del pensiero antico. Studi e testi, 79: un volume sull'allegoresi filoniana, continuazione del mio Allegoria, I, è in pubblicazione a Milano: Allegoria, II, Filone, ac. di F. CALABI, con ricca documentazione cui rinvio.

81 Clemente, Strom. V 14, 138, 2, conosce e cita anche un frammento di Metrodoro (fr. 37 Koerte), discepolo di Epicuro, che compare come la decima delle Sentenze Vaticane (Gnomologium Epicureum Vaticanum, ed. M. Marcovich in calce a Diogenis Laertii Vitae philosophorum, I, Lipsiae 1999): "Ricordati che, esendo mortale per natura e avendo ricevuto un tempo determinato, ti sei inoltrato nelle indagini sulla natura, sulla infinità e sull'eternità, e hai veduto 'le cose che sono, saranno e già sono state' (lliade, I 70)". Cosi Clemente: “Ma chi vuole ascolti anche Metrodoro, che, sebbene epicureo, per lo meno qui ha parlato divinamente: 'Ricordati, o Menestrato, che tu sei nato mortale e hai ricevuto una vita limitata, ma, salito con l'anima fino all'eternità e all'infinità delle cose, tu hai visto le cose che saranno e che furono'».

82 Uno studio sistematico delle fonti di Clemente, per certi versi ancora insuperato, è quello di $\mathrm{J}$. Gabrielsson, Über die Quelle des Clemens Alexandrinus, I-II, Upsala 1906-1909. 
attivi ad Alessandria e contemporanei, in relazione soprattutto alle origini della filosofia, del ruolo dei barbari e di quello dei Greci nella nascita della sapienza, della valutazione stessa della cultura greca: Clemente procede proprio in questa prospettiva. Era in corso una discussione sulla vera sapienza che coinvolgeva pagani (di tradizione greca) e cristiani (di tradizione giudaica, ma decisi a rapportarsi alla cultura greca e a trarne beneficio, quando non a respingerla in toto, almeno nelle dichiarazioni) ${ }^{83}$; abbiamo accennato al coinvolgimento anche di Taziano. Evidentemente, sia Diogene sia Clemente sia Taziano stesso partecipano a questo infervorato clima culturale, in cui la cultura cristiana, ancora in fieri, decideva la posizione da assumere nei confronti dell'eredità classica.

${ }_{83}$ Questo dibattito è molto ben illustrato, nelle sue ascendenze e nei suoi sviluppi, da BoYs-STONES, Post-Hellenistic Philosophy, pp. 76-98; 151-202: ne discuto anch'io nella parte finale del mio Mysterion negli Stromateis, cit. 